\title{
Application of Tsai in the calibration of badminton Hawkeye system based on computer vision
}

\author{
$\mathrm{Li} \mathrm{Li}^{1, \mathrm{a}}$ \\ ${ }^{1}$ Physical Education Department of Guangxi University, China \\ aili807@foxmail.com
}

Keywords: badminton Hawkeye system, Tsai, calibration, computer vision

\begin{abstract}
Camera calibration is the necessary step of a computer vision system. The Tsai two-stage technique is used to calibrate the badminton Hawkeye system in this paper. The internal and the external parameters and the transformation relation of the two cameras are obtained after calibrated, which lay the foundation for the obtaining of 3-D information.
\end{abstract}

\section{Introduction}

With the development of badminton in the direction of more rapid and accurate, a higher requirement is put forward to the touch judge. The disputation of the sideline is well solved after the application of the badminton Hawkeye system.

Generally, the internal and external parameters of the cameras could be obtained from camera calibration. The main job of the badminton Hawkeye system is to obtain the 3-D information based on the 2-D images, then the three dimensional reconstruction is performed to judge whether the placement of badminton is in or out. Camera calibration is the necessary step, so it has very important practical significant to study the camera calibration to the badminton Hawkeye system.

The calibration method based on radial constraint is presented by Tsai ${ }^{[1]}$ in 1986 . Most of the equations are linear in the radial constraint method, which makes the difficulty of solving the equations greatly reduce, fast and time saving. The Tsai two-stage technique is divided into two steps: firstly, the external parameter of camera is obtained according to least square method to solve the overdetermined linear equations; secondly, the internal parameter of camera is solved according to whether the camera is under the consideration of distortion.

However, Tsai only considered the radial distortion, without tangential distortion. RegG.Willson ${ }^{[2]}$ calibrated the fixed cameras with $\mathrm{C}$ programming language base on Tsai model, which promoted the application of Tsai two-stage technique. The tangential distortion was considered by Weng ${ }^{[3-4]}$ et al based on Tsai two-stage technique, and the corresponding algorithm was proposed.

\section{Camera Model}

\section{Parameters of Camera.}

The internal optical properties and geometrical features including image center, focal length, lens distortion et al are described by camera internal parameters. External parameters is the 3D position and direction of the camera coordinates relative to the world coordinate. 
Table1 Parameters of Camera model

\begin{tabular}{c|c}
\hline Parameters & expression \\
\hline perspective transformation & $A=\left[\begin{array}{ccc}\alpha_{x} & \gamma & u_{0} \\
0 & \alpha_{y} & v_{0} \\
0 & 0 & 1\end{array}\right]$ \\
\hline radial and tangential distortion & $\mathrm{k}_{1}, \mathrm{k}_{2}, \mathrm{p}_{1}, \mathrm{p}_{2}$ \\
\hline External parameters & $R=\left[\begin{array}{ccc}r_{1} & r_{2} & r_{3} \\
r_{4} & r_{5} & r_{6} \\
r_{7} & r_{8} & r_{9}\end{array}\right] T=\left[\begin{array}{c}t_{x} \\
t_{y} \\
t_{z}\end{array}\right]$
\end{tabular}

Where $\alpha_{x}, \alpha_{y}, u_{0}, v_{0}, \gamma$ is the internal parameter of linear model, $\alpha_{x}, \alpha_{y}$ is focal length, $u_{0}$, $v_{0}$ is optical center; $\gamma=0 . \mathrm{R}$ is rotation matrix, $\mathrm{T}$ is translation matrix, $\mathrm{k}_{1}, \mathrm{k}_{2}$ is radial parameter, $\mathrm{p}_{1}$, $\mathrm{p}_{2}$ is tangential distortion.

Camera Model with radial distortion

The camera model with considering the length radial distortion is shown in fig.1. $\mathrm{P}\left(\mathrm{X}_{w}, \mathrm{Y}_{w}, \mathrm{Z}_{w}\right)$ is the $3 \mathrm{D}$ space point of the mass point of the badminton; $\left(\mathrm{X}_{c}, \mathrm{Y}_{c}, \mathrm{Z}_{c}\right)$ is the coordinates of the mass point in the camera coordinate; $\left(X_{u}, Y_{u}\right)$ is the corresponding image coordinates of the space point $\mathrm{P}$ in the ideal camera model; $\left(\mathrm{X}_{d}, \mathrm{Y}_{d}\right)$ is the actual images coordinates caused by the length radial distortion.

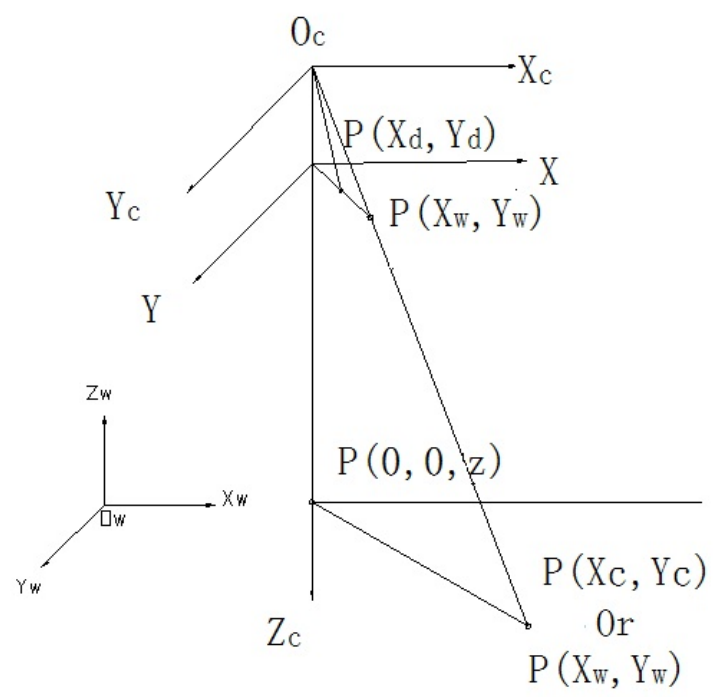

Fig. 1 The Camera model with radial distortion

Homogeneous coordinates of world coordinate and camera coordinate of a space point $\mathrm{P}$ could be presented by $\left(X_{w}, Y_{w}, Z_{w}, 1\right)^{T}$ and $\left(X_{c}, Y_{c}, Z_{c}, 1\right)^{T}$, there are the following relations:

$$
\left[\begin{array}{c}
X_{c} \\
Y_{c} \\
Z_{c} \\
1
\end{array}\right]=\left[\begin{array}{cc}
R & T \\
O^{T} & 1
\end{array}\right]\left[\begin{array}{c}
X_{w} \\
Y_{w} \\
Z_{w} \\
1
\end{array}\right]=M_{1}\left[\begin{array}{c}
X_{w} \\
Y_{w} \\
Z_{w} \\
1
\end{array}\right]
$$


Where $R=\left[\begin{array}{lll}r_{1} & r_{2} & r_{3} \\ r_{4} & r_{5} & r_{6} \\ r_{7} & r_{8} & r_{9}\end{array}\right], T=\left[\begin{array}{c}t_{x} \\ t_{y} \\ t_{z}\end{array}\right], O=\left[\begin{array}{lll}0 & 0 & 0\end{array}\right]^{T}$.

According equation (1), there are the following relations:

$$
\left\{\begin{array}{l}
X_{c}=r_{1} X_{w}+r_{2} Y_{w}+r_{3} Z_{w}+T_{x} \\
Y_{c}=r_{4} X_{w}+r_{5} Y_{w}+r_{6} Z_{w}+T_{y} \\
Z_{c}=r_{7} X_{w}+r_{8} Y_{w}+r_{9} Z_{w}+T_{z}
\end{array}\right.
$$

According the radial alignment constraint:

$$
\frac{X_{c}}{Y_{c}}=\frac{X_{d}}{Y_{d}}=\frac{X_{c}=r_{1} X_{w}+r_{2} Y_{w}+r_{3} Z_{w}+T_{x}}{r_{4} X_{w}+r_{5} Y_{w}+r_{6} Z_{w}+T_{y}}
$$

Equation (3) is transposed to:

$$
X_{w} Y_{d} \frac{r_{1}}{T_{y}}+Y_{w} Y_{d} \frac{r_{2}}{T_{y}}+Z_{w} Y_{d} \frac{r_{3}}{T_{y}}+Y_{d} \frac{T_{x}}{T_{y}}-X_{w} X_{d} \frac{r_{4}}{T_{y}}-Y_{w} X_{d} \frac{r_{5}}{T_{y}}-Z_{w} X_{d} \frac{r_{6}}{T_{y}}=X_{d}
$$

Equation (4) is expressed by vectors as below:

$$
\begin{aligned}
& {\left[\begin{array}{lllllll}
X_{w} Y_{d} & Y_{w} Y_{d} & Z_{w} Y_{d} & Y_{d} & -X_{w} X_{d} & -Y_{w} X_{d} & -Z_{w} X_{d}
\end{array}\right] \bullet} \\
& {\left[\begin{array}{lllllll}
\frac{r_{1}}{T_{y}} & \frac{r_{2}}{T_{y}} & \frac{r_{3}}{T_{y}} & \frac{T_{x}}{T_{y}} & \frac{r_{4}}{T_{y}} & \frac{r_{5}}{T_{y}} & \frac{r_{6}}{T_{y}}
\end{array}\right]^{T}=X_{d}}
\end{aligned}
$$

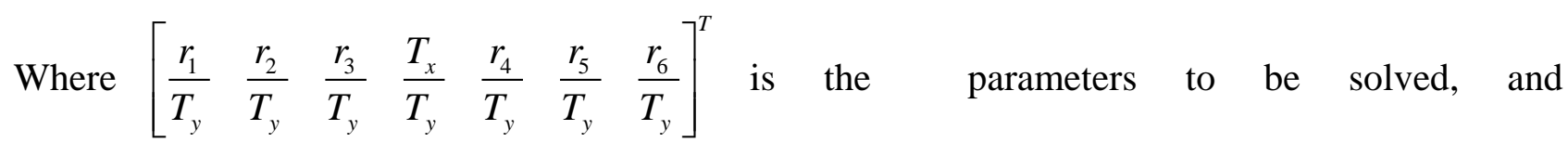
$\left[\begin{array}{lllllll}X_{w} Y_{d} & Y_{w} Y_{d} & Z_{w} Y_{d} & Y_{d} & -X_{w} X_{d} & -Y_{w} X_{d} & -Z_{w} X_{d}\end{array}\right]$ is the known parameters.

There're offset between the ideal image coordinate under the ideal camera coordinate and the actual coordinate, so the transformation relation between could be presented as:

$$
\left\{\begin{array}{c}
X_{d}=s_{x} \frac{N_{f x}}{N_{c x} d_{x}} X_{d}+u_{0} \\
Y_{d}=\frac{Y_{d}}{d_{y}}+v_{0}
\end{array}\right.
$$

Where $d_{x}$ is the pixel interval of the camera in the $\mathrm{X}$ direction, $d_{y}$ is the is the pixel interval of the camera in the Y direction; $N_{c x}$ is the pixel number of the camera in the $\mathrm{X}$ direction, $N_{f x}$ is the line pixel number that computer acquisition in the $\mathrm{X}$ direction; $\left(u_{0}, \mathrm{v}_{0}\right)$ is the optical center of camera; $s_{x}$ is the transformation scale factor of the image.

\section{The process of calibration}

For the scalding board as shown in fig.2, we could find that all the squares are in the black rectangular area, so internal part of the scalding board could be separated from the background by threshold segmentation. The center coordinate of the squares could be obtained by edge extraction after separating the scalding board. Multiple images were continuous taken to scald for more accurate results. The transformation relation between the coordinate of A camera and B camera could be solved after obtaining the internal and external parameters of the camera. Because of the cameras of the badminton Hawkeye system in this paper are fixed, scalding the camera once after the system is determined. The program flow is shown in fig.3. 


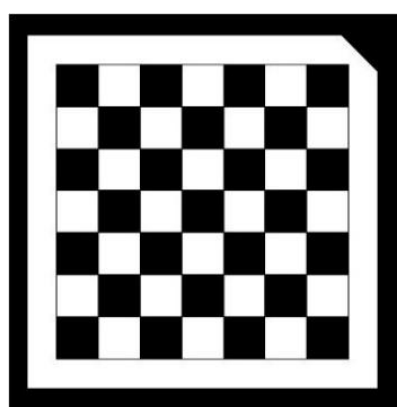

Fig.2 Calibration Board

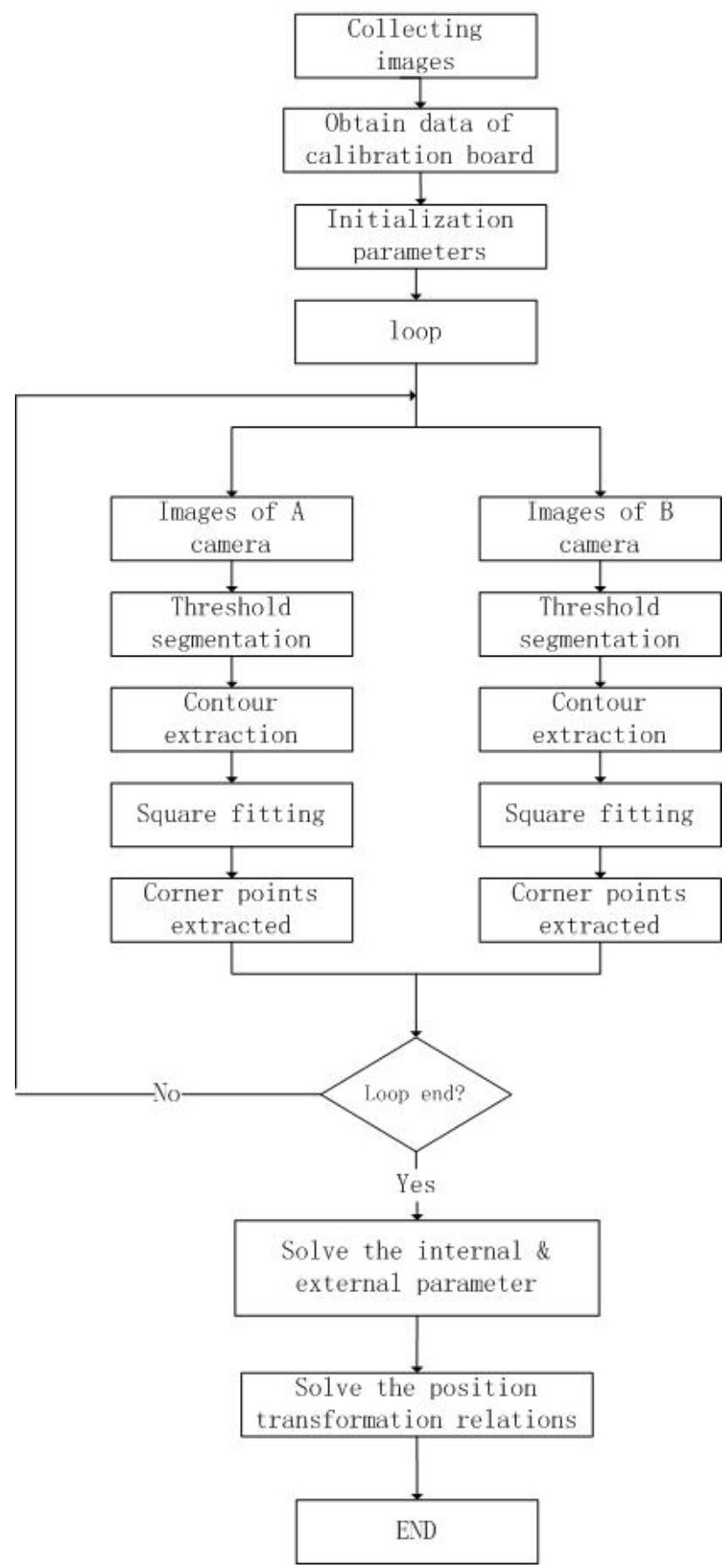

Fig.3 The calibrating flowchart of Binocular Vision System

\section{Results and analysis of calibration}

The scalding program is developed by hdevelop interface of HALCON. Firstly, the original data 
of the scalding board is obtained bycaltab_ points function; secondly, the square area of the scalding board in the image is found by find_caltab function; and then, the center coordinate of the squares are found by find_marks_and _ pose function, then the coordinate value was save in the data array; last, the transformation relation between internal and external parameters of the cameras is obtained. The calibration results of the system is shown as fig.4.

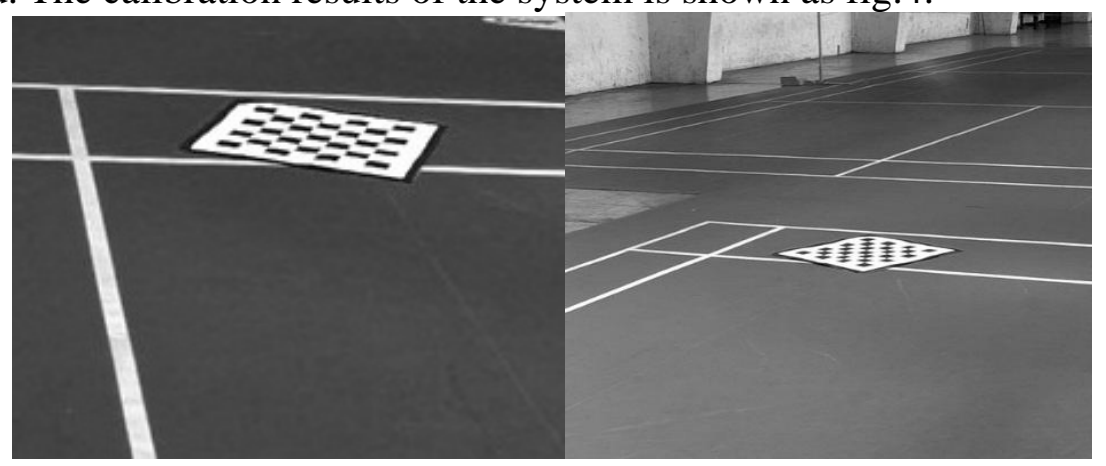

(a)

(b)

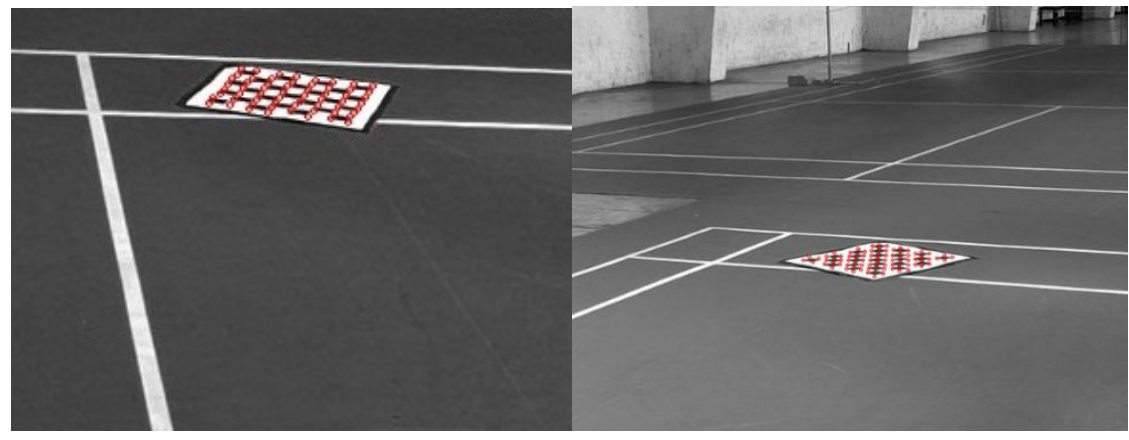

(c)

(d)

Fig.4 The calibration results of badminton Hawkeye system

Where (a) and (b) are the collected images of A and B camera of the system; (c) and (d) are the images after calibrating. The scalding board is placed in different place and angle for higher calibration accuracy. Fig. 4 is just one of them. The calibration result of the world coordinate is shown as fig. 5 with the same method.

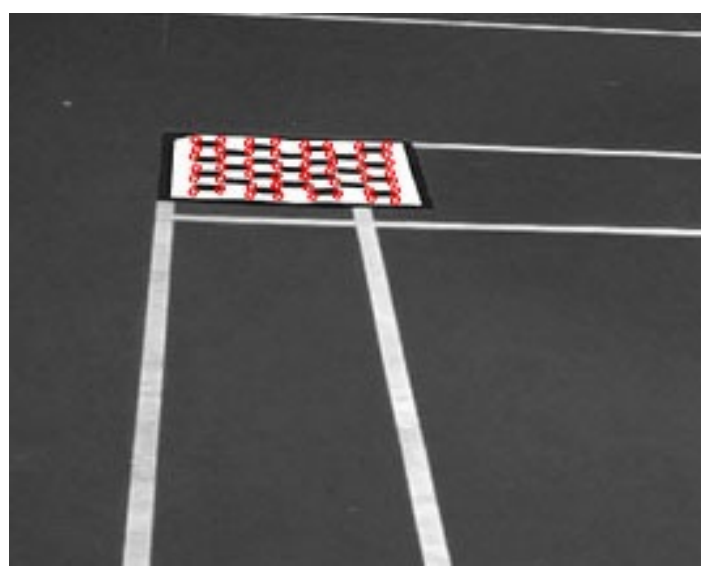

Fig. 5 The calibration result of World Coordinate

\section{References}

[1] Tsai R.Y. An efficient and accurate camera calibration technique for 3D machine vision. Proc. CVPR'86. New York: IEEE.1986.364-374

[2] W. Faig. Calibration of close-range photogrammetry system: Mathematical Formulation. 
Photogrammetric Eng. Remote Sensing, 1975, 41(12):1479-1486

[3] R.K. Lenz and Tsai R.Y. Techniques for calibration of the scale factor and image center for high accuracy 3D machine vision metrology. IEEE Trans. On PAMI, 1998,10(5):713-720

[4] Qiu.ML, Ma SD. Overview of camera calibration for computer vision. Acta Auto Atica Sinica,2000,26(1):43-55

[5] Yang XF, Huang YM. New corner detection algorithm of chessboard image for camera calibration. Chinese Journal of Scientific instruments. 2011,32(5):1109-1113

[6] Xu Y, Zhou J, Zhou YH. On Stereo Matching Technology. Computer Engineering and Applications, 2003.15:1-6

[7] Abdel-Aziz Y.I, Karara H.M. direct linear transformation from comparator coordinates into object space coordinates in close-range photogrammetry. In U. of Illinois Symposium on Close-range Photogrammetry, Urbana: University of Illinois at Urbana-Champaign, 1971.1-18

[8] Qin XW, Wen ZF, Qiao WW. Image processing based on OpenCV. Electronic Test.2011,7:39-41 